\title{
Health Economic Studies on Complementary and Integrative Medicine
}

\author{
Claudia M. Witt \\ Institut für Sozialmedizin, Epidemiologie und Gesundheitsökonomie, Charité, Universitätsmedizin Berlin, Germany
}

Over the last few years health economic data have become increasingly relevant for decision makers faced with the challenge of reconciling the growing demand for healthcare services with the funds available $[1,2]$.

The UK and Germany may serve as examples to highlight this increasing relevance. The National Institute of Health and Clinical Excellence (NICE) was established in 1999 to inform trusts within the National Health Service of England and Wales. It uses cost-effectiveness analysis to guide the allocation of resources across preventative and curative interventions [3]. In general, if a treatment costs more than GBP 20,000-30,000 per additional quality-adjusted life year (QALY), it is not considered cost-effective [4]. In 2004 the Institut für Qualität und Wirtschaftlichkeit im Gesundheitswesen (IQWiG) was established in Germany to provide information on reimbursement and pricing policies for the statutory health insurance funds. It uses a different approach and evaluates competing technologies within specific therapeutic areas, placing more weight on clinical evidence and the relative efficiency of the competing therapies [3]. This new approach has received some criticism [5], but also some support [3]. The difference is that, whereas NICE is guiding resource allocation across an entire system, IQWiG has responsibility within the bounds of a particular therapeutic area. In this editorial I will focus on the more common approach of cost-effectiveness analysis for resource allocation across an entire system.

When deciding on how to allocate limited financial resources in healthcare, the core question is: What do we want to pay for? At first glance this seems obvious, namely a costeffective treatment for a given condition. However, in reality it is often difficult to generalize the results from economic studies, partly due to methodological aspects, but also due to the influence of the underlying study setting (e.g. the healthcare system itself).

\section{KARGER}

Fax +497614520714

Information@Karger.de

www.karger.com (c) 2011 S. Karger GmbH, Freiburg

$1661-4119 / 11 / 0181-0006 \$ 38.00 / 0$

Accessible online at:

www.karger.com/fok

\section{Types of Economic Evaluations}

Although there are multiple uses for cost estimates in healthcare research and policy making, the majority of evaluations fall into two broad categories [6]: i) assessment of the aggregated economic burden of disease and ii) economic evaluations of specific healthcare interventions or programs. For complementary and integrative medicine, economic evaluations of interventions play a more prominent role, and there are three different types: i) cost of disease analysis, ii) costbenefit analysis and iii) cost-effectiveness analysis. Cost-utility and cost minimization analyses are subcategories of the costeffectiveness analysis (see table 1 for the key difference between the various types of economic evaluation).

\section{Cost Data}

Some cost data have to be available in order to proceed with economic evaluations. This could be retrieved directly from the health insurance companies or from treatment records and patient questionnaires. There are different types of costs: direct, indirect and intangible costs. Direct costs include those resources directly arising from the provision of healthcare, such as the time spent by healthcare professionals, medicines and equipment. Indirect costs represent the impact of illness and treatment on the ability to work and on leisure time. Intangible costs are difficult to measure - they take into account the pain and suffering that result from undergoing a treatment - and due to this are often not included. Cost components are included according to the perspective of the economic analysis. The societal perspective is the most comprehensive approach. The patients' perspective includes all costs relevant from the viewpoint of the patient. In an analysis conducted from a health services perspective, some direct and indirect 
Table 1. Types of economic analysis

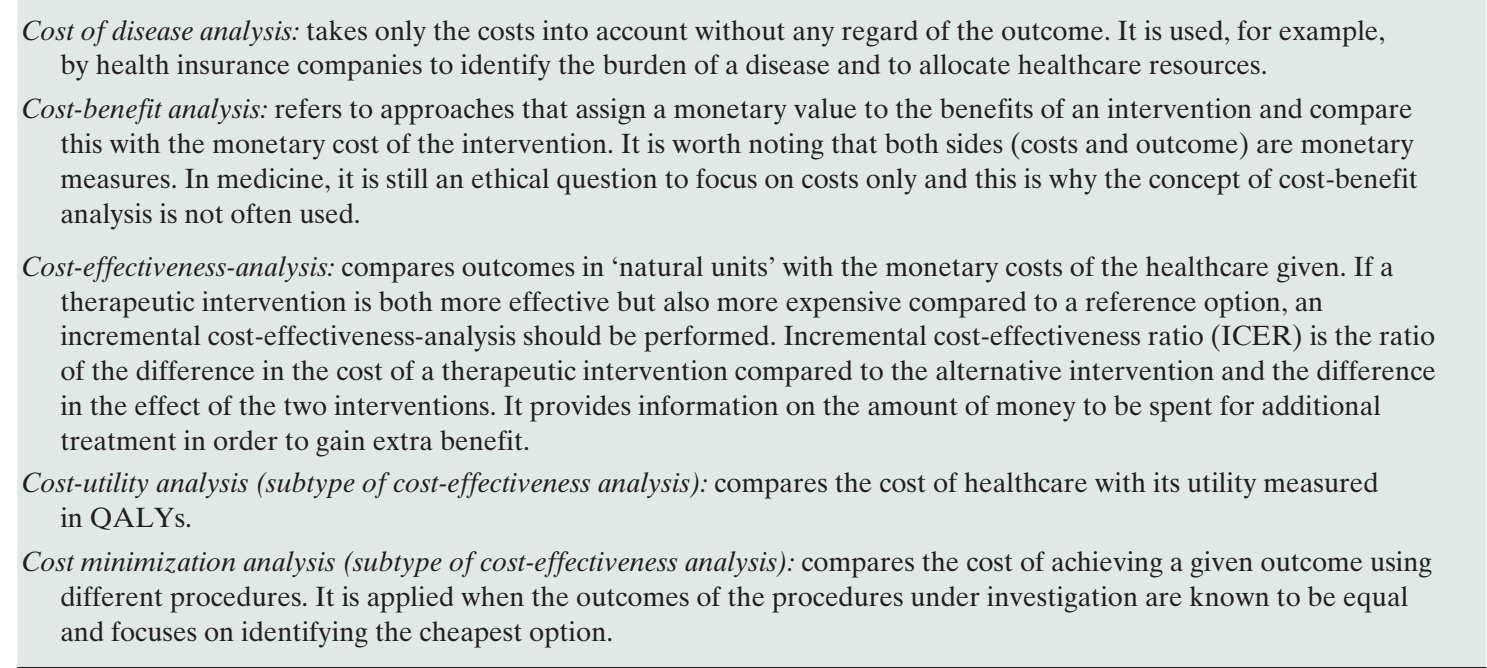

Cost of disease analysis: takes only the costs into account without any regard of the outcome. It is used, for example, by health insurance companies to identify the burden of a disease and to allocate healthcare resources. measures. In medicine, it is still an ethical question to focus on costs only and this is why the concept of cost-benefit therapeutic intervention is both more effective but also more expensive compared to a reference option, an incremental cost-effectiveness-analysis should be performed. Incremental cost-effectiveness ratio (ICER) is the ratio of the difference in the cost of a therapeutic intervention compared to the alternative intervention and the difference in the effect of the two interventions. It provides information on the amount of money to be spent for additional

Cost-utility analysis (subtype of cost-effectiveness analysis): compares the cost of healthcare with its utility measure in QALYs. different procedures. It is applied when the outcomes of the procedures under investigation are known to be equal and focuses on identifying the cheapest option.

costs which are not reimbursed are excluded. Furthermore, a cost analysis may only be disease-specific or it may have a wider focus and include all cost data (overall costs) for a patient independent of the diagnosis. For a disease-specific cost analysis, all costs that are directly associated with the disease under investigation have to be identified. If a study is longer than 12 months, the costs and effects have to be discounted. Discounting is a technique which allows the calculation of the present value of future costs or future effects of healthcare interventions. The discounting rate, which is used in economic evaluations to discount the future health gains and future costs, varies for each country.

\section{Data on Outcomes}

Outcomes from treatments can be measured in 'natural units' (e.g. $\mathrm{mm} \mathrm{Hg}$ measuring systolic blood pressure or $\mathrm{mm}$ on a pain visual analogue scale) or more complex measures such as quality of life. Although one treatment may help someone to live longer, it may also have serious side effects. Another treatment may not help someone to live as long, but it may improve the quality of life of patients, for example by reducing their pain or disability (e.g. palliative care). QALYs are a 'utility' measure and combine both the aspects of length and quality of life and represent the overall outcome in a more comprehensive way [4].

\section{Economic Evaluations from Complementary and Integrative Medicine}

The number of economic evaluations on complementary and integrative medicine has increased over the last few years, and the largest number has been done for acupuncture either in addition or compared to routine care. The first comprehensive cost-effectiveness study on acupuncture came from the UK and included patients with headache [7], followed by a further UK study including patients with chronic low back pain [8] and studies from Germany on chronic low back pain [9], chronic neck pain [10], osteoarthritis of the hip and the knee [11], headache [12], dysmenorrhea [13] and allergic rhinitis [14]. All of those evaluations used a cost-utility analysis approach. The studies from the UK based their cost values on patient-reported use of resources, whereas the German studies used data provided by the health insurance companies. Interestingly, the results from both countries were comparable even though the healthcare systems in the UK and Germany are quite different.

\section{Limitations of Economic Evaluations}

The underlying assumption in health economic studies is that the resources available, to society as a whole, are limited and that decisions have to be made about their best use. This means that the decision to fund interventions which have shown a greater benefit than the control but have higher costs, does depend on a willingness of society to pay a certain amount more for a certain additional benefit. This cut-off point or threshold for cost-effectiveness is not only a methodological but also an ethical issue. From an ethical point of view, there is an implication that some patients will be refused or not offered treatment for the sake of other patients. However, in reality such choices are indeed being made all the time. It is challenging methodologically as there is substantial variation in both the data and the methods for healthcare cost evaluation across different studies. This applies even to studies with a seemingly similar objective. It is thus important to understand the context and the methods of each study. In addition, it is important to note that the results of cost-effectiveness analysis are valid mainly for the country where the study was performed. They are based on the respective national 
Table 2. Relevant aspects of health economic evaluations [16]
1. Study question: Was a well-defined question posed in an answerable form and does it have health economic relevance?

2. Selection of alternatives: The alternative interventions should be described in sufficient detail and the rationale of the alternatives for comparison should be given.

3. Form of evaluation: form of evaluation with a clear justification as to why this form was chosen.

4. Effectiveness data: For a single effectiveness study, details of the design and the results of that study should be given. For a meta-analysis, an overview of a number of effectiveness studies, methods of synthesis should be given.

5. Benefit measurement and valuation: The primary outcome measure(s) for the economic evaluation should be clearly stated. If health benefits have been valued, details should be given of the methods used. If changes in productivity (indirect benefits) are included, they should be reported separately and their relevance to the study question discussed.

6. Costing: Quantities of resources should be reported separately from the prices (unit costs) of those resources. Methods for the estimation of both quantities and prices (unit costs) should be given. The currency and price data should be recorded and details of any adjustment for inflation, or currency conversion, given.

7. Modeling: Details should be given of any modeling used in the economic study and key parameters should be justified.

8. Adjustment for timing of costs and benefits: The time horizon over which costs and benefits are considered should be given. The discount rate(s) should be given and the choice of rate(s) justified. If costs or benefits are not discounted, an explanation should be given.

9. Allowance for uncertainty: Details should be given of the statistical tests performed and the confidence intervals around the main variables. For sensitivity analysis, details should be given as well as justification for the choice of variables for the sensitivity analysis and the ranges over which they are varied.

10. Presentations of results: An incremental analysis should be reported, comparing the relevant alternatives. Major outcomes should be presented in a disaggregated as well as aggregated form. Any comparisons with other healthcare interventions should be made only when close similarity in study methods and settings can be demonstrated. The answer to the original study question should be given; any conclusions should follow clearly from the data reported and should be accompanied by appropriate qualifications or reservations. healthcare system, the local costs of the treatments as well as the assumptions on which the analysis is based; thus such studies may vary considerably between countries [15]. Therefore, comparing the results from different studies performed in different healthcare systems, using different approaches (e.g. different types of economic analysis or different perspectives for the costs) and comparing different treatment options, can lead to incorrect conclusions being drawn. In this respect, the field of economic analysis is more complicated than many other areas of medical research and sensitivity analyses help to provide higher quality. The aim of sensitivity analyses is to take possible scenarios into account by varying the assumptions for outcomes and costs to check the results for stability and allow more generalizability. The 10-point checklist by Drummond provides helpful guidance for the critical appraisal of economic studies (table 2) [16].
Nevertheless, the increasing relevance of results from comparative effectiveness research [17] and more acceptance of randomized pragmatic trials, which reflect routine care [18], provide a solid basis for more health economic studies in the field of complementary and integrative medicine.

\section{Conclusion}

For valid decision making on complementary and integrative medicine, more clinical and health service research is needed which includes economic data to provide realistic cost estimates for future healthcare. However, the results from economic studies have to be seen in the context of the study, and conclusions have to be drawn with caution.

\section{References}

1 Williams A: Priority setting in public and private health care. A guide through the ideological jungle. J Health Econ 1988;7:173-183.

2 Mooney G, Russel EM, Weir RD: Choices for Health Care: A Practical Introduction to the Economics of Health Care Provision. London, Macmillan, 1986.

>3 Bridges JF, Cohen JP, Grist PG, Mühlbacher AC International experience with comparative effectiveness research: Case studies from England/ Wales and Germany. Adv Health Econ Health Serv Res 2010;22:29-50.
4 Measuring effectiveness and cost effectiveness: the QALY. www.nice.org.uk/newsroom/features/ measuringeffectivenessandcosteffectivenesstheqaly.jsp.

Krauth C, John J, Aidelsburger P, Brüggenjürgen B, Hansmeier T, Hessel F, et al.: Stellungnahme der AG Methoden der gesundheitsökonomischen Evaluation (AG MEG) zu dem Methodenpapier «Methodik für die Bewertung von Verhältnissen zwischen Nutzen und Kosten im System der deutschen gesetzlichen Krankenversicherung» des Instituts für Qualität und Wirtschaftlichkeit im Gesundheitswesen (IQWiG). Gesundheitswesen 2008;70:e1-16.
6 Lipscomb J, Yabroff KR, Brown ML, Lawrence W, Barnett PG: Health care costing: data, methods, current applications. Med Care 2009;47(7 suppl 1): S1-S6.

7 Wonderling D, Vickers AJ, Grieve R, McCarney $\mathrm{R}$ : Cost effectiveness analysis of a randomised trial of acupuncture for chronic headache in primary care. BMJ 2004;328:747-749.

8 Ratcliffe J, Thomas KJ, MacPherson H, Brazier $\mathrm{J}$ : A randomised controlled trial of acupuncture care for persistent low back pain: cost effectiveness analysis. BMJ 2006;333:626. 
9 Witt CM, Jena S, Selim D, Brinkhaus B, Reinhold T, Wruck K, et al.: Pragmatic randomized trial of effectiveness and cost-effectiveness of acupuncture for chronic low back pain. Am J Epidemiol 2006; 164:487-496.

10 Willich SN, Reinhold T, Selim D, Jena S, Brinkhaus B, Witt CM: Cost-effectiveness of acupuncture treatment in patients with chronic neck pain Pain 2006;125:107-113.

11 Reinhold T, Witt CM, Jena S, Brinkhaus B, Willich SN: Quality of life and cost-effectiveness of acupuncture treatment in patients with osteoarthritis pain. Eur J Health Econ 2008;9:209-219.

12 Witt CM, Reinhold T, Jena S, Brinkhaus B, Willich $\mathrm{SN}$ : Cost-effectiveness of acupuncture treatment in patients with headache. Cephalalgia 2008;28:334345.

13 Witt CM, Reinhold T, Brinkhaus B, Roll S, Jena S, Willich SN: Acupuncture in patients with dysmenorrhea: a randomized study on clinical effectiveness and cost-effectiveness in usual care. Am J Obstet Gynecol 2008;198:166-168.

14 Witt CM, Reinhold T, Jena S, Brinkhaus B, Willich SN: Cost-effectiveness of acupuncture in women and men with allergic rhinitis: a randomized controlled study in usual care. Am J Epidemiol 2009; 169:562-571.

15 Reinhold T, Brüggenjürgen $\mathrm{B}$, Schlander M., Rosenfeld S., Hessel F, Willich SN: Economic analysis based on multinational studies: methods for adapting findings to national contexts. J Public Health 2011; 18:327-335.

16 Drummond MF, Jefferson TO: Guidelines for authors and peer reviewers of economic submissions to the BMJ. The BMJ Economic Evaluation Working Party. BMJ 1996;313:275-283.

17 Conway PH, Clancy C: Comparative-effectiveness research - implications of the Federal Coordinating Council's Report. N Engl J Med 2009;361:328-330.

18 Witt CM: Efficacy, effectiveness, pragmatic trials - guidance on terminology and the advantages of pragmatic trials. Forsch Komplementärmed Klass Naturheilkd 2009;16:292-294. 